IIIIIII Open Access Articles

\title{
Transit Search for Exoplanets around Alpha Centauri A and B with ASTERIA
}

The MIT Faculty has made this article openly available. Please share how this access benefits you. Your story matters.

\begin{tabular}{|l|l|}
\hline As Published & $10.3847 / 1538-3881 /$ abf2c0 \\
\hline Publisher & American Astronomical Society \\
\hline \hline Version & Final published version \\
\hline Citable link & https://hdl.handle.net/1721.1/135652 \\
\hline Terms of Use & $\begin{array}{l}\text { Article is made available in accordance with the publisher's } \\
\text { policy and may be subject to US copyright law. Please refer to the } \\
\text { publisher's site for terms of use. }\end{array}$ \\
\hline
\end{tabular}




\title{
Transit Search for Exoplanets around Alpha Centauri A and B with ASTERIA
}

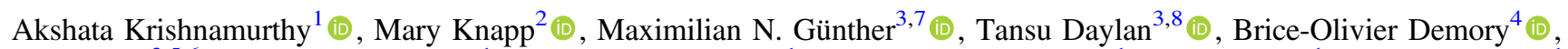

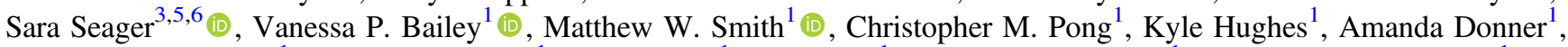 \\ Peter Di Pasquale ${ }^{1}$, Brian Campuzano ${ }^{1}$, Colin Smith ${ }^{1}$, Jason Luu ${ }^{1}$, Alessandra Babuscia ${ }^{1}$, Robert L. Bocchino, Jr. ${ }^{1}$, \\ Jessica Loveland ${ }^{1}$, Cody Colley ${ }^{1}$, Tobias Gedenk ${ }^{2}$, Tejas Kulkarni ${ }^{1}$, Mary White ${ }^{1}$, Joel Krajewski ${ }^{1}$, and Lorraine Fesq ${ }^{1}$ \\ ${ }^{1}$ Jet Propulsion Laboratory, California Institute of Technology, 4800 Oak Grove Drive, Pasadena, CA 91109, USA; akshata.krishnamurthy@jpl.nasa.gov \\ ${ }^{2}$ Haystack Observatory, Massachusetts Institute of Technology, Cambridge, MA 02139, USA \\ ${ }^{3}$ Department of Physics and Kavli Institute for Astrophysics and Space Research, Massachusetts Institute of Technology, Cambridge, MA 02139, USA \\ ${ }^{4}$ Center for Space and Habitability, University of Bern, Gesellschaftsstrasse 6, CH-3012 Bern, Switzerland \\ ${ }^{5}$ Department of Earth, Atmospheric, and Planetary Sciences, Massachusetts Institute of Technology, 77 Massachusetts Avenue, Cambridge, MA 02139, USA \\ ${ }^{6}$ Department of Aeronautics and Astronautics, Massachusetts Institute of Technology, Cambridge, MA 02139, USA \\ Received 2020 September 1; revised 2021 March 25; accepted 2021 March 27; published 2021 May 21
}

\begin{abstract}
Alpha Centauri is a triple star system with two Sun-like stars, $\alpha$ Cen A $(V=0.01)$ and B $(V=1.33)$, and a third fainter red dwarf star, Proxima Centauri. Most current transit missions cannot produce precision photometry of $\alpha$ Cen A and B as their detectors saturate for these very bright stars. The Arcsecond Space Telescope Enabling Research in Astrophysics (ASTERIA) was a technology demonstration mission that successfully demonstrated two key technologies necessary for precision photometry achieving line-of-sight fine-pointing stability of $0.5^{\prime \prime} \mathrm{rms}$ and focal plane temperature control of $\pm 0.01 \mathrm{~K}$ over a period of 20 minutes. The payload consisted of a $6.7 \mathrm{~cm}$ aperture diameter refractive camera and used a scientific complementary metal-oxide semiconductor detector that enabled monitoring of the brightest stars without saturating. We obtained spatially unresolved (blended) observations of $\alpha$ Cen A and B during opportunistic science campaigns as part of ASTERIA's extended mission. The resulting $1 \sigma$ photometric precision for the blended $\alpha$ Cen A and B data is $250 \mathrm{ppm}$ (parts per million) per $9 \mathrm{~s}$ exposure. We do not find evidence of transits in the blended data. We establish limits for transiting exoplanets around both $\alpha$ Cen A and B using transit signal injection and recovery tests. We find that ASTERIA is sensitive to planets with radii as small as $3.0 R_{\oplus}$ around $\alpha$ Cen $\mathrm{A}$ and $3.7 R_{\oplus}$ around $\alpha$ Cen B, corresponding to signals of $\sim 500$ ppm (signal-tonoise ratio $=5.0$ ) in the blended data, with periods ranging from 0.5 to 6 days.
\end{abstract}

Unified Astronomy Thesaurus concepts: Exoplanets (498); Transit photometry (1709); Space telescopes (1547)

\section{Introduction}

The $\alpha$ Cen triple system, at a distance of $1.34 \mathrm{pc}$ (Pourbaix \& Boffin 2016), contains our closest Sun-like neighbors ( $\alpha$ Cen A and B; Table 1) and the low-mass star Proxima Cen. Simulations have shown that semimajor axis regions of long-term dynamical stability exist within several astronomical units of both A and B (Quarles \& Lissauer 2016, 2018). Although no planets have been confirmed around either $\alpha$ Cen A or B, a discovery or confirmation of one or more planets orbiting either star would open up unique opportunities for detailed characterization.

$\alpha$ Cen A and B have been the subject of a number of exoplanet searches with both radial velocity (RV) and transit techniques, and several planet candidates have been proposed. A spurious RV signal with a 3.236 day period, originally interpreted as a $\sim 1 M_{\oplus}$ planet orbiting $\alpha$ Cen B, was reported by Dumusque et al. (2012), although subsequent studies have identified the signal as an artifact (Hatzes 2013; Rajpaul et al. 2015). Another planet candidate transiting $\alpha$ Cen B was proposed by Demory et al. (2015), based on the potential detection of a single transit event with HST/STIS. The candidate transit had a depth of $91 \pm 12 \mathrm{ppm}\left(\sim 0.9 R_{\oplus}\right)$ and a duration of $0.150 \pm 0.007$ days. Given the transit duration, the authors placed a $2 \sigma$ upper limit on the orbital period of 20.4 days. The Transiting Exoplanet Survey Satellite (TESS; Ricker et al. 2015) observed $\alpha$ Cen AB in Sector 11. However, the target was affected by strong saturation and blooming; it is not yet clear

\footnotetext{
7 Juan Carlos Torres Fellow.

8 Kavli Fellow.
}

whether TESS data could achieve the photometric precision necessary to detect any planet transits of these two stars.

Long-term RV monitoring of each of $\alpha$ Cen A and B with CHIRON and HARPS has placed upper limits on planets with periods ranging from 2 to 1000 days (Zhao et al. 2017). The authors calculated detection limits for both white-noise (optimistic) and red-noise (pessimistic) models. For $\alpha$ Cen A, the detection limits were roughly $7-10 M_{\oplus}$ for 2 day periods and $10-20 M_{\oplus}$ for 10 day periods. For $\alpha$ Cen $\mathrm{B}$, the detection limits were roughly $1.5-3 M_{\oplus}$ for 2 day periods and $2.5-5 M_{\oplus}$ for 10 day periods. The spurious RV signal from Dumusque et al. (2012) and the photometric signal from Demory et al. (2015), both for $\alpha$ Cen B, would have been just below these RV detection limits, assuming a rocky planet with a $50 \% \mathrm{Fe}$ mass fraction (Zeng et al. 2016). A short-period planet with $100 \%$ Fe mass fraction, at $\sim 2 M_{\oplus}$ (Zeng et al. 2016), may have been detectable, depending on whether the white or red RV noise model of Zhao et al. (2017) was correct. Note that the long-term nature of the HARPS and CHIRON programs also allowed Zhao et al. (2017) to derive detection limits for habitable zone planets (500-1000 days for A, 200-500 days for B). Their detection limits for planets in the habitable zones of A and $\mathrm{B}$ were $\sim 50 M_{\oplus}$ and $\sim 10 M_{\oplus}$, respectively.

In this paper, we present a search for small transiting planets on short periods ( $\lesssim 20$ days) around $\alpha$ Cen A and B with the Arcsecond Space Telescope Enabling Research in Astrophysics (ASTERIA) CubeSat. ${ }^{9}$ Note that $\alpha$ Cen A and B are blended

\footnotetext{
9 Proxima Cen was not observed, as it is well below ASTERIA's $V \lesssim 7$ magnitude limit.
} 
Table 1

Stellar Properties of the $\alpha$ Cen System

\begin{tabular}{|c|c|c|}
\hline Property & Value & References \\
\hline R.A. (J2000) & 143936.494 & 1 \\
\hline Decl. (J2000) & -605002.37 & 1 \\
\hline Barycenter Parallax (mas) & $747.17 \pm 0.61$ & 2 \\
\hline Semimajor axis (au) & $23.55 \pm 0.02$ & 2 \\
\hline Inclination $\left(^{\circ}\right)$ & $79.320 \pm 0.011$ & 2 \\
\hline \multicolumn{3}{|c|}{$\alpha$ Cen A } \\
\hline$B(\mathrm{mag})$ & 0.72 & 3 \\
\hline$V(\mathrm{mag})$ & 0.01 & 3 \\
\hline$J(\mathrm{mag})$ & -1.15 & 3 \\
\hline ASTERIA (mag) & -0.33 & this work \\
\hline$M_{A}\left(M_{\odot}\right)$ & $1.133 \pm 0.025$ & 4 \\
\hline$R_{\star}\left(R_{\odot}\right)$ & $1.224 \pm 0.015$ & 4 \\
\hline Spectral Type & $\mathrm{G} 2 \mathrm{~V}$ & 5 \\
\hline$T_{\text {eff }}(\mathrm{K})$ & $5766 \pm 25$ & 6 \\
\hline Limb-darkening Coefficients, $u_{1}, u_{2}$ & $0.3685,0.5069$ & 7 \\
\hline \multicolumn{3}{|c|}{$\alpha$ Cen B } \\
\hline$B(\mathrm{mag})$ & 2.21 & 3 \\
\hline$V(\mathrm{mag})$ & 1.33 & 3 \\
\hline$J(\mathrm{mag})$ & -0.01 & 3 \\
\hline ASTERIA (mag) & 0.90 & this work \\
\hline$M_{B}\left(M_{\odot}\right)$ & $0.934 \pm 0.030$ & 4 \\
\hline$R_{\star}\left(R_{\odot}\right)$ & $0.863 \pm 0.025$ & 4 \\
\hline Spectral Type & K1V & 5 \\
\hline$T_{\text {eff }}(\mathrm{K})$ & $5218 \pm 25$ & 6 \\
\hline Limb-darkening Coefficients, $u_{1}, u_{2}$ & $0.3622,0.3078$ & 7 \\
\hline
\end{tabular}

Note. See also Zhao et al. (2017) for a comprehensive review of the parameters.

References. (1) Hipparcos (van Leeuwen 2007). (2) Kervella et al. (2016). (3) Ducati (2002). (4) Kervella et al. (2003). (5) Torres et al. (2010). (6) Zhao et al. (2017). (7) TIC (Stassun et al. 2019).

(not spatially resolved) in ASTERIA data. The remainder of this paper is as follows. Sections 2 and 3 describe the ASTERIA mission and $\alpha$ Cen observations. Section 4 describes data processing, transit search, and injection and recovery methods. We present results and next steps in Sections 5 and 6, and summarize in Section 7.

\section{ASTERIA}

ASTERIA was a technology demonstration mission with the goal of demonstrating in a CubeSat platform two key technologies necessary for precision photometry: precision pointing control and precision detector thermal control (Smith et al. 2010; Pong et al. 2013). The $6 \mathrm{U}$ CubeSat $(23.9 \mathrm{~cm} \times$ $11.6 \mathrm{~cm} \times 36.6 \mathrm{~cm}, 10.2 \mathrm{~kg}$ ) was successfully launched from the International Space Station (ISS) in 2017 August and deployed into a low-Earth orbit in 2017 November. It met its technology demonstration objectives during the prime mission, with a line-of-sight fine-pointing stability of $0.5^{\prime \prime} \mathrm{rms}$ and focal plane temperature control of $\pm 0.01 \mathrm{~K}$, both over a period of 20 minutes (Pong 2018; Smith et al. 2018).

During its extended mission, ASTERIA performed opportunistic $^{10}$ science observations of known and potential

\footnotetext{
10 ASTERIA's science campaigns are called "opportunistic" because the observations were interleaved with technology demonstration exercises to support other mission objectives; ASTERIA was a technology demonstration mission, not a science mission.
}

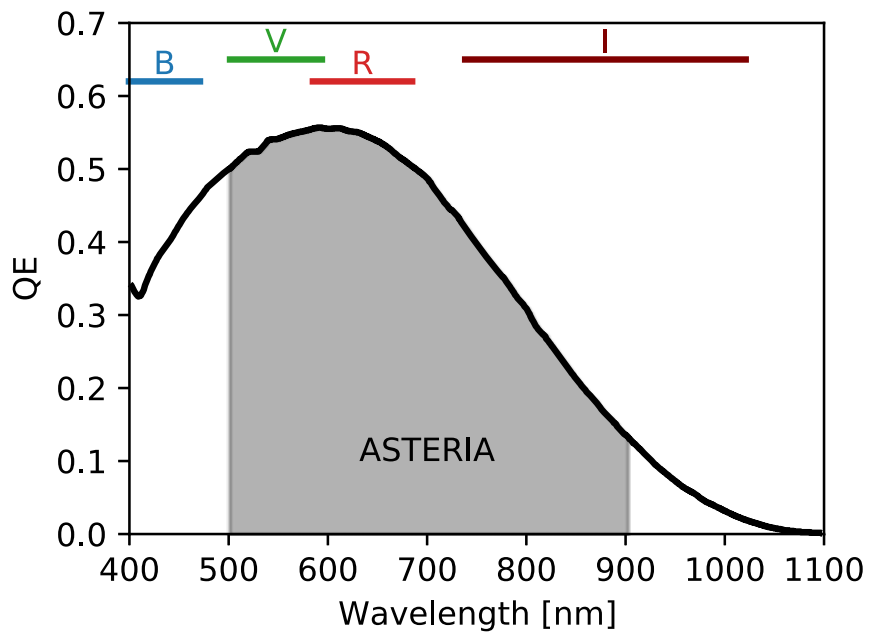

Figure 1. ASTERIA detector quantum efficiency (black line) and bandpass (500-900 nm, shaded region). Johnson $B, V, R$, and $I$ bandpass FWHMs are shown for context.

transiting exoplanet systems, including $\alpha$ Cen $\mathrm{AB}$, $55 \mathrm{Cnc}$ (Knapp et al. 2020), and HD 219134 (Seager et al. 2021). ASTERIA is the smallest telescope to detect the transit of 55 Cancri e, a super-Earth transiting a Sun-sized star (Knapp et al. 2020). ASTERIA was operational for two years until 2019 December and reentered Earth's atmosphere in 2020 April.

The ASTERIA payload was a $6.7 \mathrm{~cm}$ diameter aperture telescope, consisting of a refractive optic assembly $(f / 1.4$, $85 \mathrm{~mm}$ ), a scientific complementary metal-oxide semiconductor (sCMOS) image sensor, a two-axis piezoelectric nanopositioner, a thermal strap, and a baffle. The first lens optic had a $500-900 \mathrm{~nm}$ bandpass coating. The detector was a frontilluminated Fairchild Imaging scientific CMOS (sCMOS) detector containing $2592 \times 2192$ pixels with a pixel area of $6.5 \mu \mathrm{m}^{2}$. Figure 1 illustrates the detector quantum efficiency curve and bandpass. The plate scale was $15.8^{\prime \prime}$ per pixel, the field of view was $11.2^{\circ} \times 9.6^{\circ}$, and the telescope was intentionally defocused to produce a donut-shaped, oversampled point-spread function with a typical radius of about 10-14 pixels. With a separation of less than $6^{\prime \prime}, \alpha$ Cen A and B are entirely blended (spatially unresolved) in our data.

\section{Observations}

ASTERIA's orbit was approximately 90 minutes long, providing opportunities to perform observations during Earth eclipse, which lasted 20-30 minutes. The observations obtained are thus noncontinuous. For more details on ASTERIA's operational constraints, see Knapp et al. (2020).

We observed $\alpha$ Cen over a total of 331 orbits over four opportunistic science campaigns when the viewing geometry was favorable. The first campaign lasted from 2018 June 15 to 25 , and observations were named "so32" and "so36" (where "so" stands for science observation). The second campaign lasted from 2018 August 24 to 26 and was called "so42." The third campaign was the longest and most continuous, lasting for a full month from 2019 February 4 to 2019 March 8. This campaign was specifically intended to collect data sufficient to search for a recurrence of the Demory et al. (2015) single transit event. The observations were called "so51," "so52," "so53," “so54," “so55," "so56," “so57," “so58," “so59," "so60," "so61," "so62," and "so63." The so58 observation 
Table 2

ASTERIA $\alpha$ Cen Observations Used in the Light Curve Shown in Figure 4

\begin{tabular}{|c|c|c|c|c|c|c|}
\hline $\begin{array}{l}\text { Observation } \\
\text { Name }\end{array}$ & $\begin{array}{l}\text { First Frame } \\
\text { (UTC) }\end{array}$ & $\begin{array}{l}\text { Last Frame } \\
\text { (UTC) }\end{array}$ & $\begin{array}{l}\text { Number of } \\
\text { Orbits }\end{array}$ & $\begin{array}{l}\text { Number of } \\
\text { Images }\end{array}$ & $\begin{array}{l}\text { Integration Time (int) } \\
\text { (s) }\end{array}$ & $\begin{array}{c}\mathrm{rms} \\
(\mathrm{ppm} / \mathrm{int})\end{array}$ \\
\hline $\begin{array}{l}\text { Science Observation } 32 \\
\text { (so32) }\end{array}$ & 2018-06-15 03:04:34.8 & 2018-06-15 14:14:17.9 & 6 & 140 & 9 & 273 \\
\hline $\begin{array}{l}\text { Science Observation } 36 \\
\text { (so36) }\end{array}$ & 2018-06-24 07:16:56.6 & 2018-06-25 14:26:13.2 & 19 & 474 & 9 & 313 \\
\hline $\begin{array}{l}\text { Science Observation } 42 \\
\text { (so42) }\end{array}$ & 2018-08-24 22:15:51.8 & $2018-08-26$ 22:15:47.7 & 27 & 519 & 9 & 337 \\
\hline $\begin{array}{l}\text { Science Observation } 51 \\
\text { (so51) }\end{array}$ & 2019-02-04 03:38:15.8 & 2019-02-04 14:41:02.7 & 7 & 140 & 9 & 500 \\
\hline $\begin{array}{l}\text { Science Observation } 52 \\
\text { (so52) }\end{array}$ & 2019-02-06 01:37:15.8 & 2019-02-08 06:00:30.5 & 21 & 374 & 9 & 275 \\
\hline $\begin{array}{l}\text { Science Observation } 53 \\
\text { (so53) }\end{array}$ & 2019-02-09 04:43:09.4 & 2019-02-12 09:42:32.3 & 39 & 733 & 9 & 230 \\
\hline $\begin{array}{l}\text { Science Observation } 54 \\
\text { (so54) }\end{array}$ & 2019-02-14 02:52:42.4 & 2019-02-17 10:56:03.8 & 31 & 308 & 18 & 154 \\
\hline $\begin{array}{l}\text { Science Observation } 55 \\
\text { (so55) }\end{array}$ & 2019-02-18 00:26:42.3 & 2019-02-20 14:07:12.3 & 28 & 332 & 18 & 285 \\
\hline $\begin{array}{l}\text { Science Observation } 56 \\
\text { (so56) }\end{array}$ & 2019-02-20 23:01:08.0 & 2019-02-21 13:10:41.9 & 6 & 70 & 18 & 205 \\
\hline $\begin{array}{l}\text { Science Observation } 57 \\
\text { (so57) }\end{array}$ & 2019-02-21 22:00:26.9 & 2019-02-22 12:09:59.2 & 8 & 96 & 18 & 94 \\
\hline $\begin{array}{l}\text { Science Observation } 59 \\
\text { (so59) }\end{array}$ & 2019-02-26 23:02:55.0 & 2019-02-28 12:12:32.9 & 19 & 453 & 9 & 207 \\
\hline $\begin{array}{l}\text { Science Observation } 60 \\
\text { (so60) }\end{array}$ & 2019-02-28 19:35:19.2 & 2019-03-04 08:07:21.2 & 31 & 671 & 9 & 175 \\
\hline $\begin{array}{l}\text { Science Observation } 61 \\
\text { (so61) }\end{array}$ & 2019-03-05 11:20:14.4 & 2019-03-06 04:27:43.6 & 11 & 193 & 9 & 179 \\
\hline $\begin{array}{l}\text { Science Observation } 62 \\
\text { (so62) }\end{array}$ & 2019-03-06 11:50:21.0 & 2019-03-07 00:19:02.5 & 8 & 111 & 9 & 153 \\
\hline $\begin{array}{l}\text { Science Observation } 63 \\
\text { (so63) }\end{array}$ & 2019-03-07 12:20:41.3 & 2019-03-08 00:46:26.2 & 6 & 64 & 9 & 152 \\
\hline $\begin{array}{l}\text { Experimental Observation } 30^{*} \\
\text { (ex30) }\end{array}$ & 2019-10-05 13:37:33.0 & 2019-10-07 13:19:25.5 & 34 & 595 & 9 & 391 \\
\hline $\begin{array}{l}\text { Science Observation } 69 \\
\text { (so69) }\end{array}$ & 2019-10-08 04:10:38.6 & 2019-10-10 14:31:56.2 & 30 & 786 & 9 & 193 \\
\hline
\end{tabular}

Note. Observations designated "ex" for "experimental" involved some non-standard configuration of the instrument. In the case of ex 30 , the first 34 planned observations used the standard window arrangement while the last 5 used an experimental window placement. After the technology demonstration objectives were completed, opportunistic science observations were performed and named "so" to indicate the change in focus from technology demonstration to collection of science data.

was excluded from this analysis because a spacecraft fault rendered the data unusable. The fourth and final campaign lasted from 2019 October 5 to 10 , and the observations were called "ex30" and "so69," where "ex" indicates experimental observation. The full list of observations, timeline, number of orbits, and integration time are available in Table 2.

The camera served simultaneously for guiding and science, and the fine-pointing feedback control loop operated at $20 \mathrm{~Hz}$. Full frames could not be read at this speed. Thus, eight $64 \times 64$ pixel windows were read. The locations of these windows are shown in Figure 2. One window was centered on the target star $(\alpha$ Cen $)$ and three windows were dedicated to additional bright stars. All four stars were used as guide stars for fine-pointing control as described in Section 4.1. Data downlink bandwidth was limited, so images were coadded on board to reduce data volume. In typical ASTERIA observations, 1200 images of $50 \mathrm{~ms}$ each were coadded on board to produce a 64 pixel $\times$ 64 pixel $\times 8$ window cube with an integration time of 1 minute. For photometric observations of $\alpha$ Cen, we used an integration time of $7.5 \mathrm{~ms}$ per frame to prevent charge saturation and coadded 1200 consecutive frames to produce integrations covering $9 \mathrm{~s}$. Exposures were still triggered at the standard $20 \mathrm{~Hz}$ rate; thus, each $1200 \times 7.5 \mathrm{~ms}$ frame spans 1 minute of wall-clock time, but only $9 \mathrm{~s}$ of light exposure time. All 

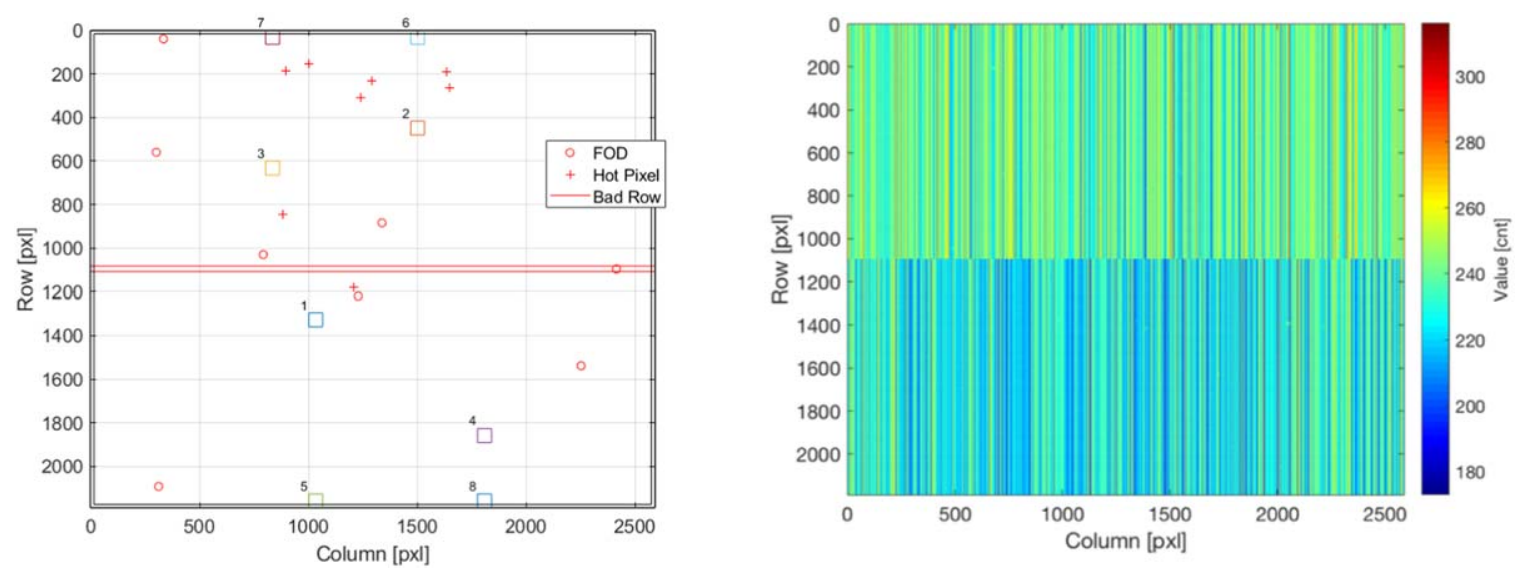

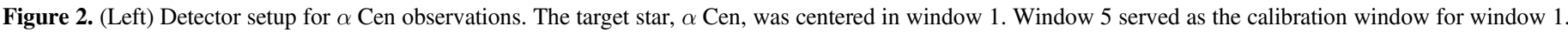

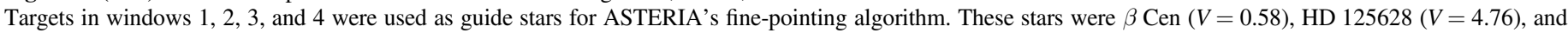

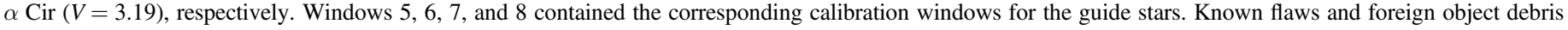

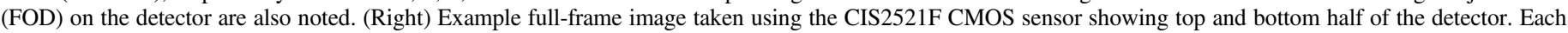
half has corresponding optically and electrically dark rows at the top and bottom of the detector that are used to calibrate bias and dark noise.

observations except so54, so55, so56, and so57 were coadded to 1 minute images before being downlinked. For the remaining observations, we coadded 2400 consecutive exposures to produce $18 \mathrm{~s}$ integration time frames spanning 2 minutes of wall-clock time, a strategy used to save onboard memory storage space during long observations in campaign three. No registration was performed prior to coadding due to the excellent fine-pointing control system that locked the target star to within $0.5^{\prime \prime}(\sim 1 / 30 \mathrm{px}) \mathrm{rms}$.

The camera was power-cycled and reinitialized at the beginning and end of each orbit. The camera was off and cold prior to each observing sequence, causing a moderate thermal transient during the first one to two orbits of the observation as the camera warmed up and the thermal control system stabilized. There is a correlation between the photometric precision and thermal housekeeping data. We investigate the efficacy of using thermal data for detrending in Section 4.

\section{Methods}

In this section, we present a summary of the $\alpha$ Cen data reduction and photometry, with details of the pipeline described in Knapp et al. (2020) and Seager et al. (2021).

\subsection{CMOS Detector and Systematics Calibration}

ASTERIA was the first space telescope mission to perform precision visible-light photometry using an sCMOS detector. sCMOS detectors provided several advantages over the widely used traditional CCD technology, which is known for its higher quantum efficiency and sensitivity. At the time of ASTERIA's launch, only sCMOS detectors were capable of the $20 \mathrm{~Hz}$ readout rate required for the fine-pointing control mode. ${ }^{11}$ With their higher frame rate, the sCMOS detector enabled the observation of very bright stars like $\alpha$ Cen without saturating. Additionally, sCMOS detectors have very low dark current even when operating at room temperature. Thus, an active cooling system was not required, making sCMOS highly suitable for compact platforms like CubeSats. A two-part active

\footnotetext{
11 Present-day frame transfer, electron-multiplying CCD sensors are capable of similarly high readout rates of $20 \mathrm{~Hz}$ and are manufactured by companies such as Teledyne e2v (Wang et al. 2020) using a back-thinning process that ensures high performance in very low-light conditions.
}

heating system was used to maintain detector thermal stability (Smith et al. 2018). For the observations described here, the focal plane temperature set point was $301 \mathrm{~K}$ and the baffle temperature set point was $291.5 \mathrm{~K}$.

The sCMOS technology was relatively new at the time of ASTERIA's launch and the systematics were not very well understood. Although CMOS technology (where chargevoltage conversion takes place in each pixel rather than a common output structure like in CCDs) has been around for a while, several limitations prevented their use in precision lowlight applications. The sCMOS detectors, a new generation of CMOS image sensors, provided higher imaging performance in low-light conditions, along with low readout noise and a wide dynamic range at extremely rapid readout rates. However, they exhibited column-dependent offset and gain variations, because each column had its own amplifier and analog-to-digital converter, and each pixel had its own amplifier. These effects could be corrected using flat frames (images with uniform illumination of the detector) of varying illumination levels $(\mathrm{Hu}$ et al. 2016). Due to the budget and schedule limitations inherent to ASTERIA's status as a technology demonstration mission, science-grade ground characterization of the detector was not performed. Hence, we did not have traditional dark, bias, and flat frames from ground testing that are typically available for space telescope missions (Fanelli et al. 2011; Vanderspek et al. 2018).

Due to the absence of a shutter, we were unable to collect on-orbit dark and bias frames. Instead, we used contemporaneous "calibration windows" to characterize noise contributions from dark and bias (for details, see A. Krishnamurthy 2021, in preparation, and Krishnamurthy 2020). Sixteen rows at the bottom and top of the detector were physically blocked from light exposure during detector fabrication. The first eight rows closest to the detector edge provided a measurement of dark current, while the next eight rows were electrically tied to ground to prevent charge accumulation and served as a good source for the estimation of bias noise. We placed a calibration window (window 5) directly below the target star window (window 1), spanning the same detector columns and capturing the electrically and optically dark rows. The location of the target, the additional guide stars, and the corresponding calibration windows are shown in Figure 2. Contribution from 
dark noise was calculated using calibration window rows 56:64 and was found to be negligible due to the short integration time of $7.5 \mathrm{~ms}$ per frame. Bias noise was calculated in the data reduction pipeline using rows 48:56 in the calibration window.

Although bias frames are typically used to correct for offsets, flat frames are used to correct column-dependent pixel-to-pixel gain variations caused due to the presence of an amplifier and ADC converter at every column and an amplifier at every pixel. We attempted to take on-orbit flat frames by illuminating the detector with scattered sunlight, following the procedure outlined in Knapp et al. (2020) and Seager et al. (2021). However, these observations failed because the camera did not initialize properly. Hence, we did not perform a flat correction for the $\alpha$ Cen observations.

We assessed the effect of nonlinearity on $\alpha$ Cen observations through analysis of flight data. The full-well capacity of the ASTERIA detector is $30,000 \mathrm{e}^{-}$and maximum nonlinearity is $2 \%$ as given in the manufacturer's data sheet (Fairchild Imaging 2014). Laboratory measurements were not performed with the flight detector to assess linearity as ASTERIA was a technology demonstration mission. For $\alpha$ Cen observations, we operated at $10,000 \mathrm{e}^{-}$(1/3 full-well capacity). Assuming maximum nonlinearity of $2 \%$, the error in transit depth calculations is $4 \mathrm{ppm}$. The effect of nonlinearity is thus minimal for relative photometry so long as the target stays fixed. Hence, we did not correct for this effect.

\subsection{Data Processing}

We began by manually reviewing all the data and discarding coadded images that had background contamination due to stray light during orbital "sunrise" or "sunset" at the beginning or end of an orbit. In a few observations, we also discarded the first one to two orbits due to thermal transients at the beginning of an observation. Next, we performed bias removal using the calibration window. We used the electrically dark rows to calculate the median bias for each column and subtracted this from each pixel in the corresponding column within the target star window. We then performed background subtraction by calculating the median of the charge within rows 0:20 for every column within the target star frame and subtracting that from every pixel in that column. These rows only contained background or sky noise and were devoid of target star flux. The raw image and output of the data processing step are shown in Figure 3.

\subsection{Aperture Photometry and Detrending}

We began by calculating the weighted first moments of flux to determine the mean $x / y$ centroid of the star. We used the coordinates of the median of centroids of all frames as input to the aperture photometry. We performed aperture photometry with exact aperture edges (i.e., the exact intersection of each pixel with the aperture is calculated, and the flux within the aperture is summed) using the CircularAperture routine within the photutils Python package (Bradley et al. 2019). We determined the optimal aperture size by comparing the standard deviation of the stellar flux time series for aperture sizes of radii ranging from 10 to 15 pixels. The contribution of the stellar flux intensity and background noise increased with an increase in aperture size. The improvement in standard deviation (i.e., photometric precision) due to an increase in stellar flux intensity was eventually overcome by the increase
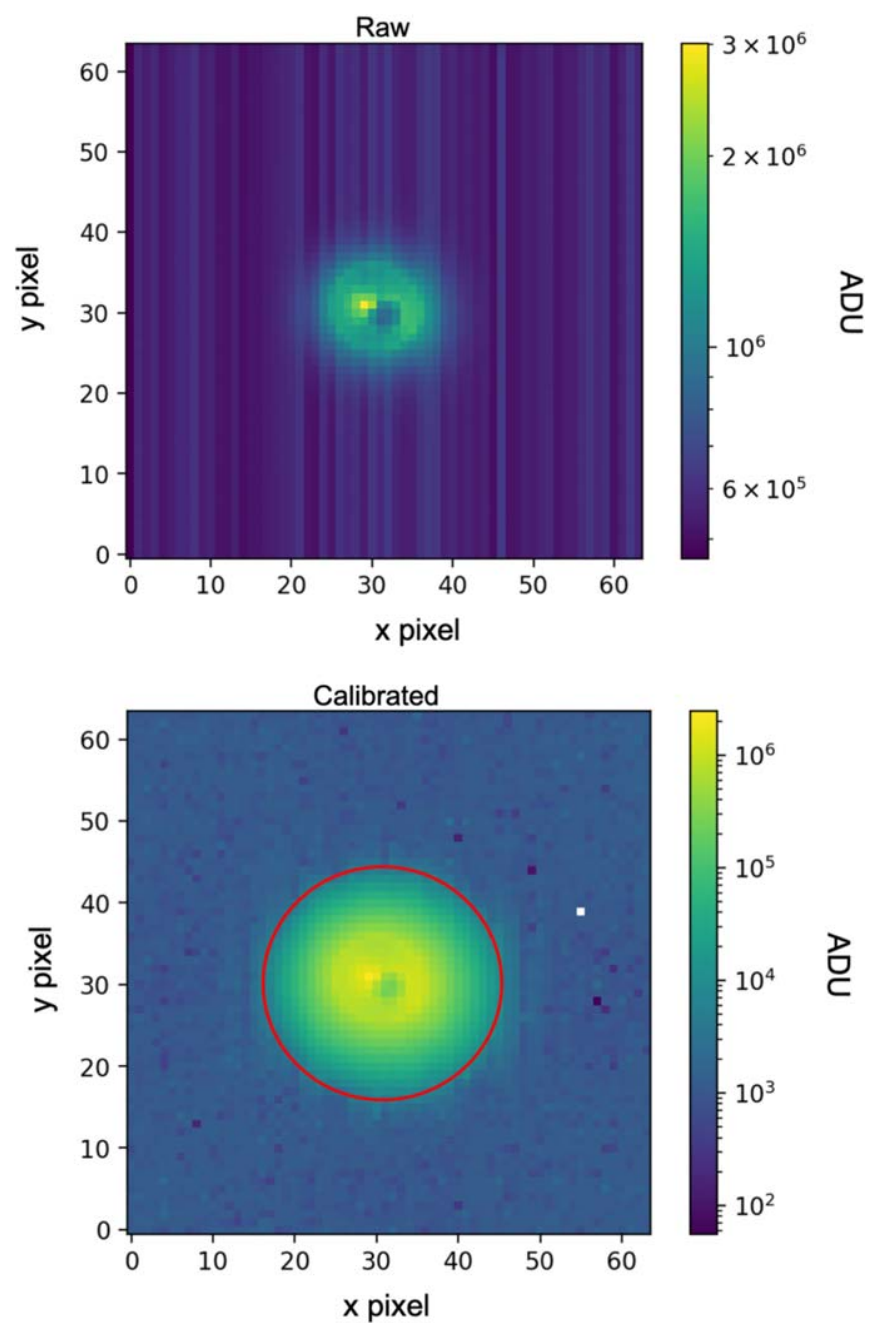

Figure 3. ASTERIA $\alpha$ Cen representative raw (top) and calibrated (bottom) image. The $x$ - and $y$-axes show the number of pixels in the image. The color bar indicates light intensity in units of analog-to-digital units (ADU). The image is a $64 \times 64$ pixel window out of a $2592 \times 2192$ pixel sCMOS detector. The target star is located in the center of the image and is intentionally defocused to minimize the effects of intrapixel gain variations and spacecraft jitter. The raw image is dominated by a fixed pattern noise caused by column-dependent gain and offset variations due to the presence of amplifiers and ADC converters along each column. We performed photometric flux extraction with a circular aperture of radius 14 pixels, shown on the calibrated image in red.

in background noise. We selected an aperture radius of 14 pixels based on the minimization of standard deviation. We then performed the photometric flux extraction for all of the $\alpha$ Cen observations.

We compared three methods for light-curve detrending: polynomial detrending against spacecraft parameters, biweight filtering, and spline smoothing. First, we tested linear, quadratic, and cubic trends of time, $x / y$ centroid position, and onboard temperatures measured at the lens housing. (Raw data and housekeeping parameters for an example observation are shown in Figure 7.). Second, we compared these results by applying a biweight filter to flatten the light curve using the wotan Python package (Hippke et al. 2019). A biweight filter is a robust location estimator whose weights depend on the distance of the residuals from the central location, making it less affected by outliers than, for example, a median filter (for details, see Mosteller \& Tukey 1977). We detrended the light curve using a biweight filter and sliding $5 \sigma$ clipping with a 


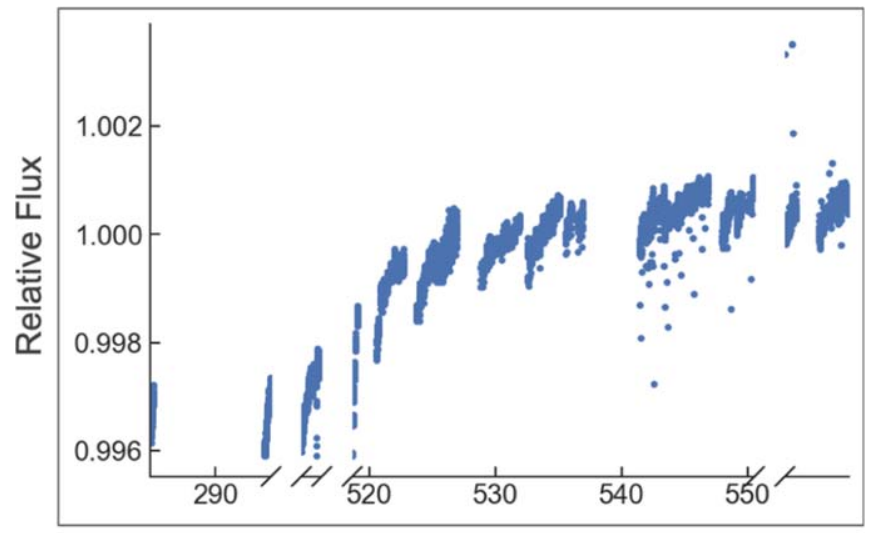

Time (BJD-2458000) days

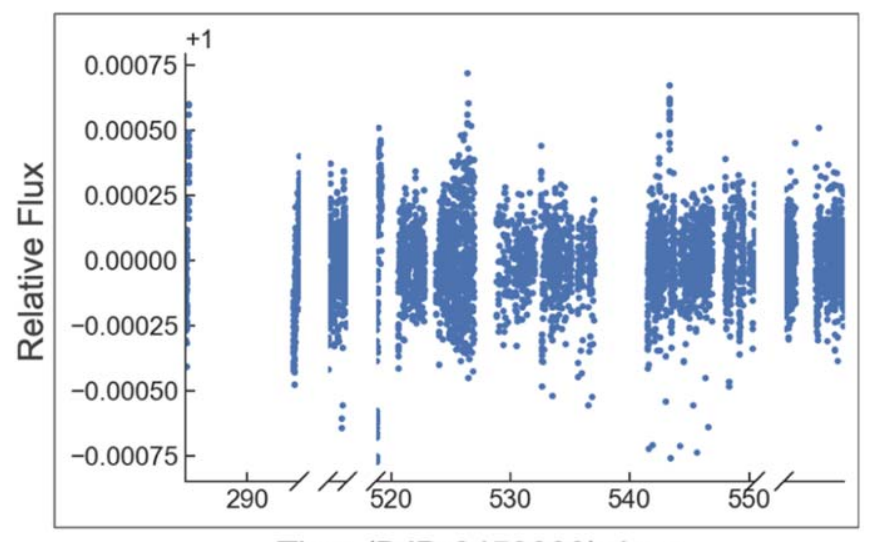

Time (BJD-2458000) days

Figure 4. The ASTERIA raw (top) and detrended (bottom) light curves for the blended $\alpha$ Cen A and B system plotted using the brokenaxes Python package to show all $\alpha$ Cen campaigns. The raw light curve is produced by performing photometric flux extraction of all calibrated $\alpha$ Cen data used in the analysis, plotted against the Barycentric Julian Date (BJD). The observations were performed as part of several opportunistic science campaigns; hence, the data set is sparsely sampled and noncontinuous. The detrended light curve was obtained via a biweight filter and sliding $5 \sigma$ clipping with 0.5 day windows using the wotan Python package.

0.5 day window (raw and biweight-filter-detrended light curves are shown in Figure 4). Third, we detrended the ASTERIA data using a spline at cubic order with a knot separation of $12 \mathrm{hr}$. The biweight filter and spline fitting methods provided consistent improvement in photometric precision (calculated using the standard deviation of the light curve) and were thus used for the transit search campaigns described in Section 4.5.

We also extracted photometric time series for the three additional guide stars ( $\beta$ Cen, HD 125628, and $\alpha$ Cir) to investigate shared systematics between the target and additional guide stars and explore the feasibility of performing relative photometry. $\beta$ Cen (window 2 ) is a variable of the $\beta$ Cep type (Samus et al. 2017), $\beta$ Cir (window 4) is a pulsating/rotational variable (Samus et al. 2017), while HD 125628 (window 3) is faint $(V=4.76)$ compared to $\alpha$ Cen. Variability was clearly visible in the light curves of $\beta$ Cen and $\alpha$ Cir, making them unsuitable for differential photometry or detrending. The light curve for HD 125628 was stable, but the photometric precision was much lower than that of $\alpha$ Cen due to the combination of very short exposure times and the large difference in $V$ magnitude between HD 125628 and $\alpha$ Cen. For these reasons, we concluded that using any of the three stars to perform differential photometry would not have yielded improvement in precision.

\section{4. $\alpha$ Cen A and B Synthetic Photometry}

The $\alpha$ Cen A and B system was unresolved in ASTERIA imaging. To calculate the deblended detection limit for each star, we calculated the relative brightness of the two stars in ASTERIA's effective passband (Section 2, Figure 1). We used the Python pysynphot package (STScI Development Team 2013) and the Pickles (1998) spectral library to calculate synthetic photometry (Table 1 ). We calculated $\Delta$ mag $\sim 1.2$. Given the uncertainties in the ASTERIA end-to-end throughput as a function of wavelength, we assumed a $\sim 5 \%$ error on this $\Delta$ mag.

\subsection{Transit Search}

We perform two transit searches: one targeted search for the spurious RV signal reported in Dumusque et al. (2012) and one blind search for transit-like signals.

We performed a test of the spurious RV signal discussed by Dumusque et al. (2012), Hatzes (2013), and Rajpaul et al. (2015) by fitting the ASTERIA data with a prior on the ephemeris based on that reported in Dumusque et al. (2012; Section 1). For this purpose, we detrended the ASTERIA data using a spline at cubic order with a knot separation of $12 \mathrm{hr}$. We then used allesfitter (Günther \& Daylan 2019, 2021) to sample from the posterior probability distribution of the light-curve model. We assumed a circular orbit and placed flat and wide priors on all orbital parameters (see Günther \& Daylan 2021 for the complete parameter list) except the orbital period and epoch, where we adopted a flat prior 10 times wider than the uncertainties reported in Dumusque et al. (2012). We performed the light-curve fit using two different background models: a flat baseline with a free offset and a Gaussian process with the Matérn 3/2 covariance having a free amplitude and timescale.

Furthermore, motivated by the single transit-like signal in Demory et al. (2015), we also performed a blind search for potential transiting planets. In this analysis, we started with a global detrending of the light curve using a biweight filter and a sliding-window, $5 \sigma$ clipping, both with a 0.5 day window using wotan (Hippke et al. 2019). We used allesfitter and the transitleastsquares (TLS) Python package (Hippke \& Heller 2019) to look for additional transit-like signals. We searched for transits down to signal-to-noise ratio $(\mathrm{S} / \mathrm{N})=5$ while simultaneously flattening the light curve using a biweight filter and sliding-window sigma clipping ( $3 \sigma$ up, $20 \sigma$ down) with windows of 1 day.

\subsection{Injection and Recovery Tests}

We performed transit injection and recovery tests to assess the completeness of our planet search. To investigate the detection rate as a function of planet size and period, we used allesfitter to inject transits generated by the ellc (Maxted 2016) Python package. We used a uniform planetary radius grid of $0.5<R_{p}\left(R_{\oplus}\right) \leqslant 22.0$ using steps of $0.1 R_{\oplus}$. For planetary period, we used a grid of $0.5<P \leqslant 30.0$ with uniform steps of 0.1 days. We performed separate tests for $\alpha$ Cen $\mathrm{A}$ and $\mathrm{B}$ using nonlinear limb darkening with coefficients listed in Table 1 and dilution factor calculated based on the estimated magnitudes of the stars, as described in 


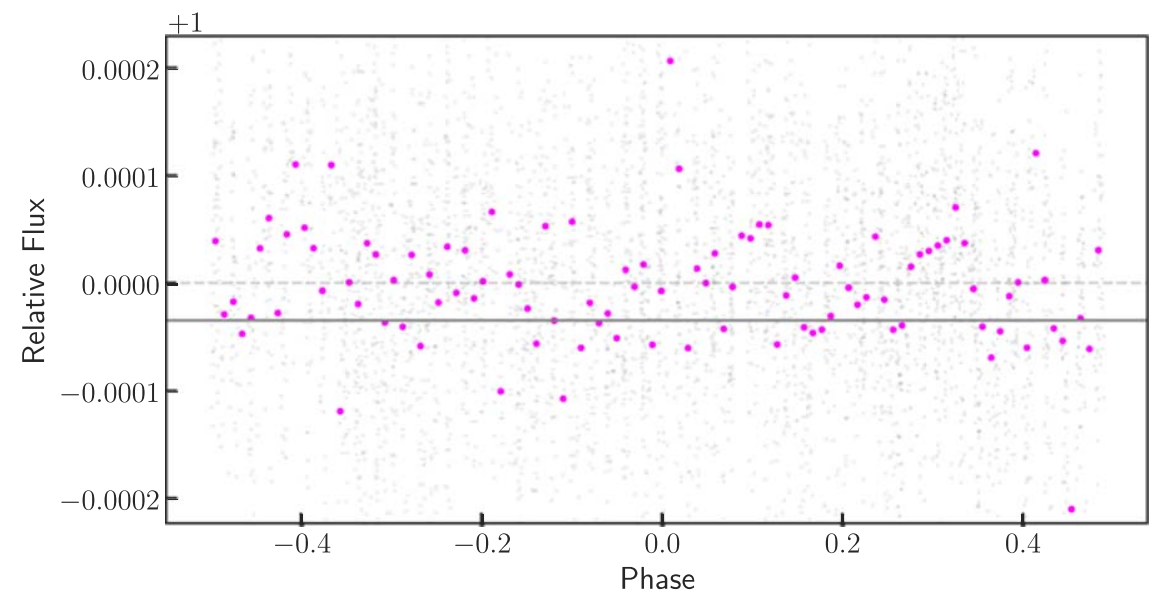

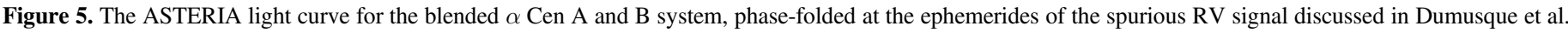

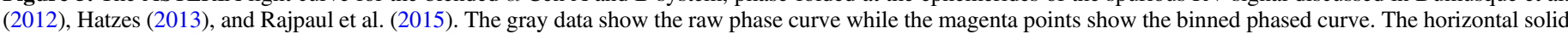

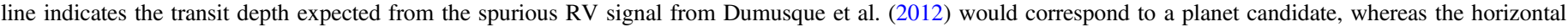
dashed line simply highlights unity for visual comparison. We do not find evidence of a photometric counterpart of the signal at a period of 3.236 days.

Section 4.4. After injection, we flattened the light curve using a biweight filter and sliding-window sigma clipping ( $3 \sigma$ up, $20 \sigma$ down) with windows of 1 day.

We then searched for transit-like signals using TLS and identified signals as "recovered" if (i) the found period is a multiple of half the injected period to within $5 \%$ and (ii) the found epoch matches the injected epoch to within $5 \%$ of the period. We estimated detection rates using an $\mathrm{S} / \mathrm{N}$ threshold between 3 and 7 , with results for $S / N=5$ presented in Section 5 .

\section{Results and Discussion}

The overall photometric precision of ASTERIA is $250 \mathrm{ppm}$ per $9 \mathrm{~s}$ exposure (ranging from $150 \mathrm{ppm}$ to $500 \mathrm{ppm}$ for various observations) for the blended system. We were neither able to attribute the residual systematic noise to known systematic effects nor remove it, due to the absence of traditional ground characterization data.

We do not find any evidence of transit signatures in the ASTERIA $\alpha$ Cen AB data, in either blind or targeted searches. Given the detection limits described below, signals such as the spurious RV signal described in Dumusque et al. (2012) and Rajpaul et al. (2015), and the photometric signal in Demory et al. (2015) are out of reach here. The resulting phase-folded light curve from the targeted search for a photometric counterpart to the spurious RV signal in Dumusque et al. (2012) is shown in Figure 5. The blind search for transiting signatures also did not find evidence of any transit-like signals.

Injection and recovery tests were performed in order to investigate the detectability of planets. The results, as presented in Figure 6, show that ASTERIA achieved a $5 \sigma$ detection limit of $\sim 500 \mathrm{ppm}$ on the blended $\alpha$ Cen A and B system for planets with periods ranging $0.5-6$ days. This corresponds to radii as small as 3.0 $R_{\oplus}$ around A and as small as $3.7 R_{\oplus}$ around B. For comparison, Jupiter, with a mass of $317.8 M_{\oplus}$, has a radius of $11.2 R_{\oplus}$, and Neptune, with a mass of $17.15 M_{\oplus}$, has a radius of $3.9 R_{\oplus}$. The regime of planets on longer orbits, as well as subNeptunes and Earth-sized planets, remains to be explored.

We place new upper limits on planets with very short periods of 0.5-2 days, namely that any hidden planets must have radii below 3.0 $R_{\oplus}$ for $\alpha$ Cen A, and below 3.7 $R_{\oplus}$ for $\alpha$ Cen B. For planets with periods of 2-6 days, we achieve comparable limits to the radial velocity study of Zhao et al. (2017) for $\alpha$ Cen A, albeit slightly higher for $\alpha \mathrm{Cen} \mathrm{B}$, due to source blending. Zhao et al. (2017) ruled out $\sim 10 M_{\oplus}$ (i.e., $3.2 R_{\oplus}$ assuming a Neptune-like density of $1.638 \mathrm{~g} \mathrm{~cm}^{-3}$ ) on periods of 2-10 days around $\alpha$ Cen A, comparable to our $3.0 R_{\oplus}$ limit. Around $\alpha$ Cen B, they rule out $\sim 3 M_{\oplus}$ (i.e., $2.2 R_{\oplus}$ assuming a Neptune-like density) planets, while we reach $3.7 R_{\oplus}$.

We also investigated detection rate as a function of observed transit depth. Despite ASTERIA's sparsely sampled data, and $\alpha$ Cen A and B being blended together, we are sensitive to $500 \mathrm{ppm}$ signals at $5 \sigma$ in the blended data and to planets as small as Neptunes in short-period orbits.

\section{Next Steps}

ASTERIA has successfully demonstrated arcsecond-level precision pointing and millikelvin thermal control. In addition, we have provided a proof of concept of the science capability which can be further improved with systematic ground characterization of the instrument prior to launch.

As a logical next step, we have proposed a constellation of small satellites to search for transiting exoplanets, specifically Earth-size planets around the brightest Sun-like stars in Earthlike orbits. The proposed constellation is currently in the concept-study phase. It will initially consist of six to ten $12 \mathrm{U}$ CubeSats, with the potential to grow to several dozen satellites. Each satellite provides the unique capability of long-duration monitoring of one Sun-like star for as long as possible under observational constraints. With continuous observations of several nonblended stars and more robust instrument systematics calibration, the ASTERIA constellation has the potential to be a pioneering Earth-analog planet hunter looking for Earthsize planets around the brightest Sun-like stars.

\section{Summary}

ASTERIA performed photometric observations of $\alpha$ Cen A and B, both Sun-like stars, as part of multiple opportunistic science campaigns during its extended mission. ASTERIA's $1 \sigma$ photometric precision for the blended $\alpha$ Cen A and B data was $250 \mathrm{ppm}$ per $9 \mathrm{~s}$ exposure. We observed residual correlated noise at timescales similar to the orbit eclipse duration 

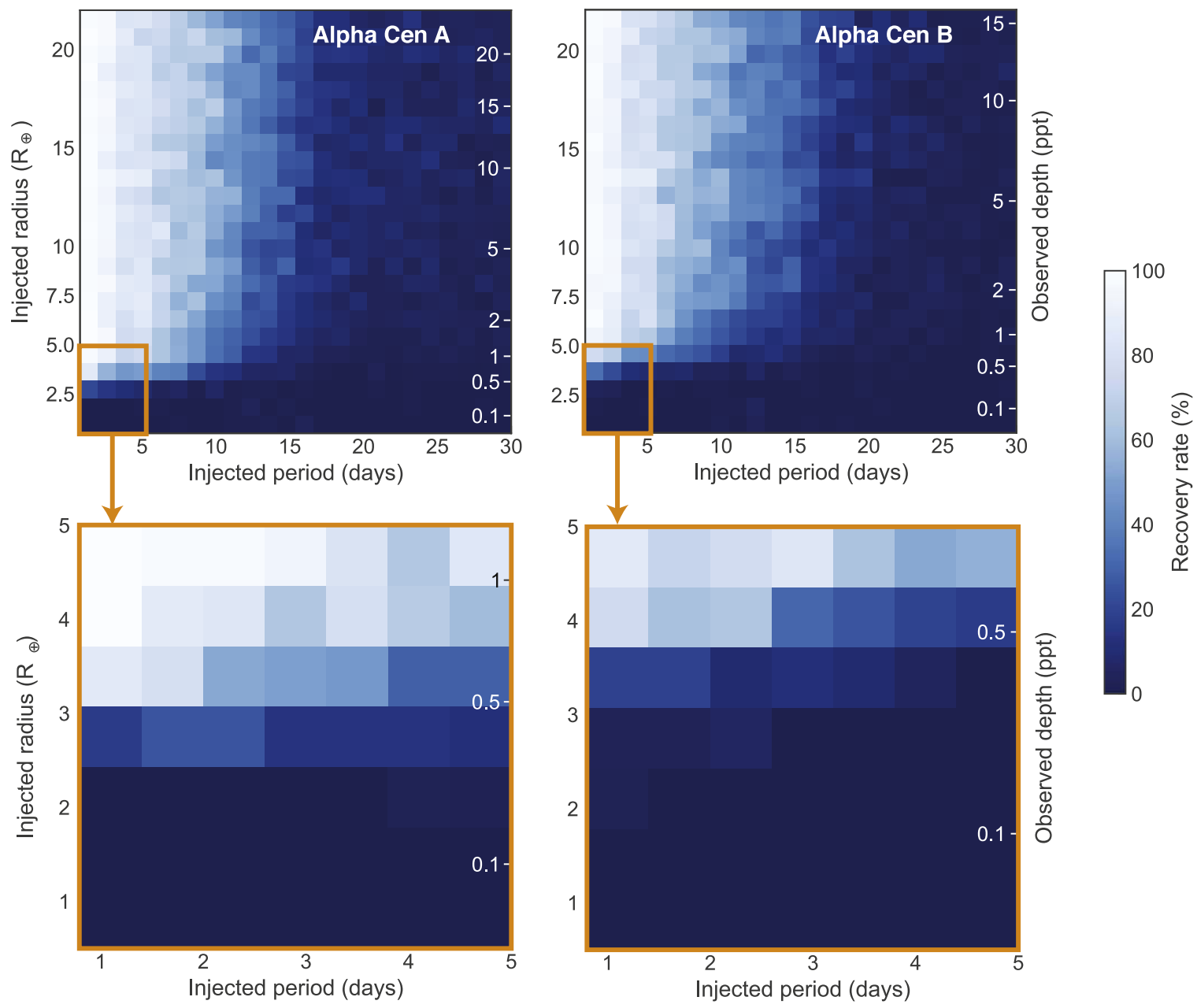

Figure 6. Results from $\alpha$ Cen A (left) and B (right) injection and recovery tests for simulated transits of planets with radii $0.5-22 R_{\oplus}$, and periods between 0.5 and 30 days. The grids represent completeness, with darker shades of blue representing lower recovery rates. The ASTERIA $\alpha$ Cen data yields near-complete recovery for Neptunes and Jupiters on orbits less than 6 days. The bottom images show zoomed-in versions of the top images that show the entire range of radii and periods used in the analysis.
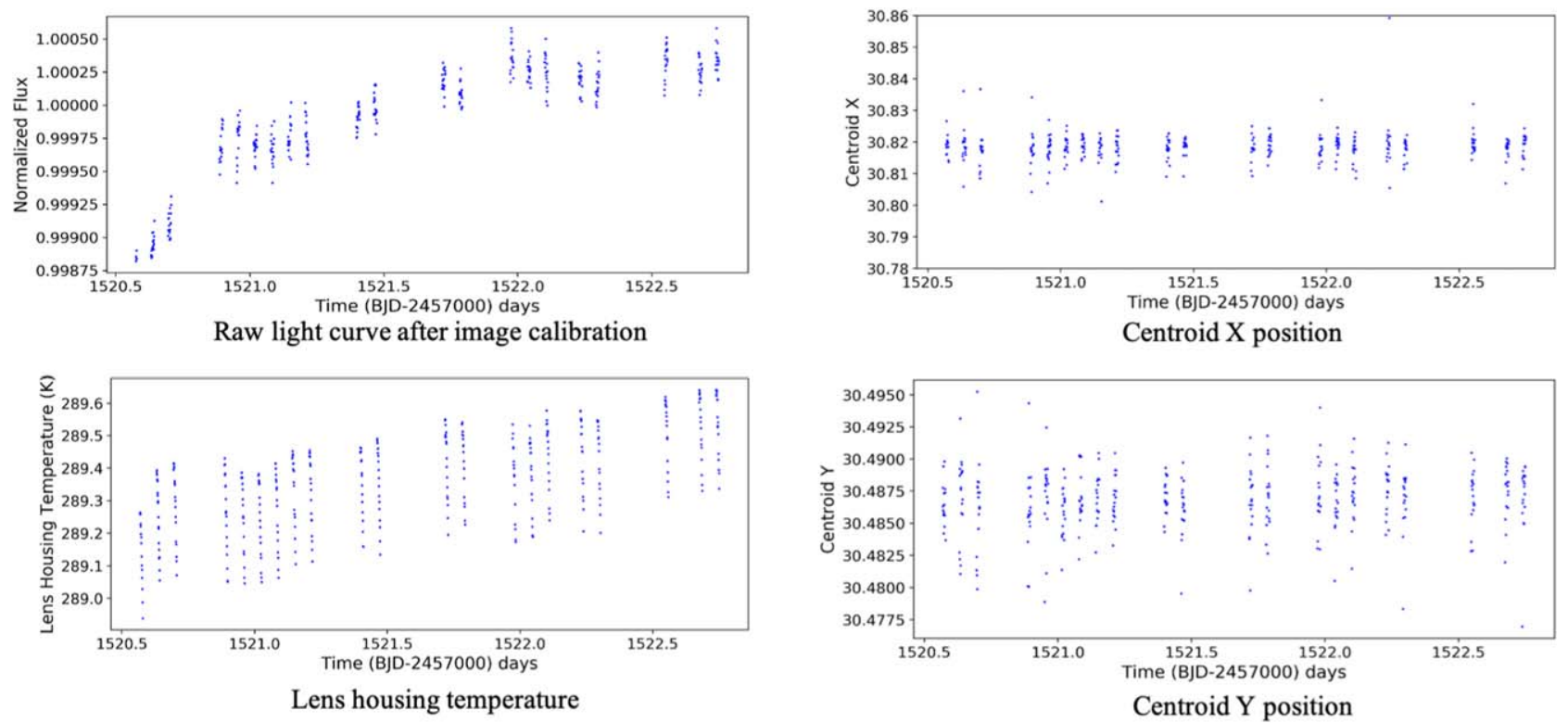

Figure 7. Raw light curve and housekeeping parameters for one observation (so69). The raw light curve (top left) is produced by performing photometric flux extraction of the calibrated $\alpha$ Cen images and is plotted against the Barycentric Julian Date (BJD). Housekeeping data including centroid position (top and bottom right) and lens housing temperature (bottom left) are shown on the same time axis as the extracted photometry. The lens housing is not under active thermal control like the detector and baffle. The lens is at a relatively lower temperature in the first orbit until the camera turns on and heats the lens housing. 
(25 minutes) that we were neither able to attribute to known systematic effects nor remove from our data.

We do not detect transit events around either $\alpha$ Cen A or B with $\mathrm{S} / \mathrm{N}>5$. We place radius limits on transiting planets around both stars using injection-recovery tests. For periods of 0.5-6 days, our resolved observations were sensitive to planets with radii as small as $3 R_{\oplus}$ around $\alpha$ Cen A and planets as small as $3.7 R_{\oplus}$ around $\alpha$ Cen $\mathrm{B}$, corresponding to signals of $\sim 500 \mathrm{ppm}(\mathrm{S} / \mathrm{N}=5.0)$ in the blended data. For the first time, we place upper limits on planets with periods of 0.5-6 days for the above-mentioned planet radii. We achieve comparable limits to the RV study of Zhao et al. (2017) on $\alpha$ Cen A and slightly higher upper limits on $\alpha$ Cen $\mathrm{B}$ for planets with periods of 2-6 days for the above-mentioned planet radii.

ASTERIA successfully demonstrated precision pointing and thermal control capabilities, which led to opportunistic highprecision photometric observations of very bright stars. The results are compelling despite the sparsely sampled data, blended target stars, and challenging reduction due to the lack of ground characterization of instrument noise. The challenges in working with unevenly sampled data with large temporal gaps point toward the need for more continuous monitoring of bright stars, perhaps through the use of a different orbit. ASTERIA's ability to monitor the brightest stars at high precision without saturating is unique among existing transit photometry missions. The results presented here provide a proof of concept for a future constellation of $12 \mathrm{U}$ satellites that would be dedicated to searching for Earth-size planets in Earthlike orbits transiting Sun-like stars (brighter than $V=6$ ). The constellation is in the concept-study phase and aims to provide a unique capability for long-duration monitoring of the brightest Sun-like stars.

We acknowledge contributions from the extended team that supported ASTERIA development, integration and test, and operations, including Len Day, Maria de Soria Santacruz-Pich, Carl Felten, Janan Ferdosi, Kristine Fong, Harrison Herzog, Jim Hofman, David Kessler, Roger Klemm, Jules Lee, Jason Munger, Lori Moore, Esha Murty, Chris Shelton, David Sternberg, Rob Sweet, Kerry Wahl, Jacqueline Weiler, Thomas Werne, Shannon Zareh, and Ansel Rothstein-Dowden. We also recognize the JPL line organization and technical mentors for the expertise they provided throughout the project.

The research was carried out in part at the Jet Propulsion Laboratory, California Institute of Technology, under a contract with the National Aeronautics and Space Administration (80NM0018D0004). M.N.G. acknowledges support from MIT's Kavli Institute as a Juan Carlos Torres Fellow. T.D. acknowledges support from MIT's Kavli Institute as a Kavli postdoctoral fellow.

We thank the JPL program management, especially Sarah Gavit and Pat Beauchamp, who oversaw ASTERIA within the Engineering and Science Directorate at JPL. We also thank Daniel Coulter and Leslie Livesay for their support.

We would also like to thank the DSS-17 ground station team at Morehead State University (MSU) in Kentucky. We acknowledge the outstanding effort of the student operators, technical staff, and program management at Morehead State University, including Chloe Hart, Sarah Wilczewski, Alex Roberts, Maria Lemaster, Lacy Wallace, Rebecca Mikula, Bob Kroll, Michael Combs, and Benjamin Malphrus.
This research has made use of NASA's Astrophysics Data System Bibliographic Services; the SIMBAD database, operated at CDS, Strasbourg, France; and the NASA Exoplanet Archive, which is operated by the California Institute of Technology, under contract with NASA under the Exoplanet Exploration Program.

Facility: ASTERIA.

Software: Numpy, Scipy, astropy (The Astropy Collaboration et al. 2013), astroquery (Astropy 2018), allesfitter (Günther \& Daylan 2019, 2021), photutils (Bradley et al. 2019), ellc (Maxted 2016), pysynphot (STScI Development Team 2013), pyBJD (https://github.com/ tboudreaux/pyBJD), matplotlib (Hunter 2007), IDL, STK, MATLAB.

\section{ORCID iDs}

Akshata Krishnamurthy (1) https://orcid.org/0000-00028781-2743

Mary Knapp (iD https://orcid.org/0000-0002-5318-7660 Maximilian N. Günther (iD https://orcid.org/0000-00023164-9086

Tansu Daylan (1D https://orcid.org/0000-0002-6939-9211

Brice-Olivier Demory (1) https://orcid.org/0000-0002-

9355-5165

Sara Seager (10 https://orcid.org/0000-0002-6892-6948

Vanessa P. Bailey (iD https://orcid.org/0000-0002-5407-2806

Matthew W. Smith (DD https://orcid.org/0000-0003-0103-8820

\section{References}

Astropy 2018, astropy/astroquery: v0.3.8 release, doi:10.5281/zenodo. 1160627

Bradley, L., Sipocz, B., Robitaille, T., et al. 2019, Astropy/photutils: v0.6, Zenodo, doi:10.5281/zenodo.2533376

Demory, B. O., Ehrenreich, D., Queloz, D., et al. 2015, MNRAS, 450, 2043

Ducati, J. R. 2002, yCat, 2237, 0

Dumusque, X., Pepe, F., Lovis, C., et al. 2012, Natur, 491, 207

Fairchild Imaging 2014, CIS2521F CMOS Image Sensor Datasheet, https:// www.fairchildimaging.com/products/scmos-sensors/cis2521

Fanelli, M., Jenkins, J., Bryson, S., et al. 2011, Kepler Data Processing Handbook (Mountain View, CA: NASA Ames Research Center)

Günther, M. N., \& Daylan, T. 2019, Allesfitter: Flexible Star and Exoplanet Inference From Photometry and Radial Velocity, Astrophysics Source Code Library, ascl:1903.003

Günther, M. N., \& Daylan, T. 2021, ApJS, 254, 13

Hatzes, A. P. 2013, ApJ, 770, 133

Hippke, M., David, T. J., Mulders, G. D., \& Heller, R. 2019, AJ, 158, 143

Hippke, M., \& Heller, R. 2019, A\&A, 623, A39

Hu, C., Bai, Y., \& Tang, P. 2016, ISPAr, 41, 749

Hunter, J. D. 2007, CSE, 9, 99

Kervella, P., Mignard, F., Mérand, A., \& Thévenin, F. 2016, A\&A, 594, A107

Kervella, P., Thévenin, F., Ségransan, D., et al. 2003, A\&A, 404, 1087

Knapp, M., Seager, S., Demory, B.-O., et al. 2020, AJ, 160, 23

Krishnamurthy, A. 2020, PhD thesis, Massachusetts Institute of Technology

Maxted, P. 2016, A\&A, 591, A111

Mosteller, F., \& Tukey, J. W. 1977, Data Analysis and Regression: A Second Course in Statistics (Reading, MA: Addison-Wesley)

Pickles, A. J. 1998, PASP, 110, 863

Pong, C. 2018, in 32nd Annual AIAA/USU Conf. on Small Satellites, SSC18PI-34 (Logan, UT: Utah State Univ. Libraries), 1, https://digitalcommons. usu.edu/cgi/viewcontent.cgi? article $=4173 \&$ context $=$ smallsat

Pong, C., Smith, M., Knutson, M., et al. 2013, AdAnS, 141, 1, http://hdl. handle.net/1721.1/81759

Pourbaix, D., \& Boffin, H. M. 2016, A\&A, 586, A90

Quarles, B., \& Lissauer, J. J. 2016, AJ, 151, 111

Quarles, B., \& Lissauer, J. J. 2018, AJ, 155, 130

Rajpaul, V., Aigrain, S., \& Roberts, S. 2015, MNRAS, 456, L6

Ricker, G. R., Winn, J. N., Vanderspek, R., et al. 2015, JATIS, 1, 014003 
Samus, N. N., Kazarovets, E. V., Durlevich, O. V., Kireeva, N. N., \& Pastukhova, E. N. 2017, ARep, 61, 80

Seager, S., Knapp, M., Demory, B.-O., et al. 2021, AJ, 161, 117

Smith, M. W., Donner, A., Knapp, M., et al. 2018, in 32nd Annual AIAA/ USU Conf. on Small Satellites, SSC18-I-08 (Logan, UT: Utah State Univ. Libraries), 1, https://digitalcommons.usu.edu/cgi/viewcontent.cgi?article= $4067 \&$ context $=$ smallsat

Smith, M. W., Seager, S., Pong, C. M., et al. 2010, Proc. SPIE, 7731, 773127

Stassun, K. G., Oelkers, R. J., Paegert, M., et al. 2019, AJ, 158, 138

STScI Development Team 2013, Pysynphot: Synthetic Photometry Software Package, Astrophysics Source Code Library, ascl:1303.023
The Astropy Collaboration, Robitaille, T. P., Tollerud, E. J., et al. 2013, A\&A, 558, A33

Torres, G., Andersen, J., \& Giménez, A. 2010, A\&ARv, 18, 67

van Leeuwen, F. 2007, A\&A, 474, 653

Vanderspek, R., Doty, J., Fausnaugh, M., \& Villasenor, J. 2018, TESS Instrument Handbook (Cambridge, MA: MIT Press), https://archive.stsci. edu/files/live/sites/mast/files

Wang, S.-Y., Ling, H.-H., Wang, B.-J., et al. 2020, Proc. SPIE, 11454 , $114542 \mathrm{P}$

Zeng, L., Sasselov, D. D., \& Jacobsen, S. B. 2016, ApJ, 819, 127

Zhao, L., Fischer, D. A., Brewer, J., Giguere, M., \& Rojas-Ayala, B. 2017, AJ, 155,24 